\section{PTH-195 LUBIPROSTONE TREATMENT IMPROVES CONSTIPATION AND RELATED SYMPTOMS IN PATIENTS REFRACTORY TO OTHER CONSTIPATION THERAPIES}

doi:10.1136/gutjnl-2013-304907.682

'R M Panas, ${ }^{2,}$ T Joswick, ${ }^{3 P}$ Lichtlen, 'D Panigrahi, , $R$ Ueno. 'Medical Affairs, ${ }^{2} R \& D$, Sucampo Pharmaceuticals, Bethesda, United States; ${ }^{3} R \& D ;{ }^{4}$ Sucampo Pharmaceuticals, Zug, Switzerland

Introduction Constipation affects millions globally; however, many patients are dissatisfied with currently-available laxative treatments, with most interested in access to other treatment options ${ }^{1}$. Lubiprostone ( $24 \mathrm{mcg}$ BID) has been shown to be effective and well-tolerated in patients with refractory constipation.

Methods Post-hoc analysis was conducted to evaluate whether lubiprostone provides relief in patients who are refractory or lacking adequate relief from other common constipation therapies. Pooled patient and medication data from two pivotal trials were assessed. Patients with a history of constipation despite the use of constipation medication during the 90 days prior to initiation of study medication were included. Response rates and symptomatic improvements in this subpopulation of refractory patients were analysed based on treatment assignment in the clinical trial: lubiprostone (24 mcg BID) or placebo. Analysis was performed on the whole and by the class of prior medication usage. Patients were considered full responders if they achieved $\geq 4$ spontaneous bowel movements (SBMs)/week. Patients achieving $\geq 3$ but $<4$ SBMs/week were moderate responders; those with $<3$ SBMs/ week were non-responders. Symptomatic improvements were also assessed.

Results Full response to treatment ranged from 52.8 to $67.2 \%$ across the 4 weeks of treatment, as compared to $32.3 \%$ to $47.4 \%$ for placebo patients. For patients who were moderate responders, response rates increased to $64.8-80.0 \%$ among the lubiprostone treatment group compared to only $46.6-57.9 \%$ of placebo patients. Lubiprostone produced a significant and full response over placebo, respectively, among the refractory patients who previously took contact laxatives $50.9-64.9 \%$ vs. $21.3-42.6 \%$; PEG solutions $57.5-75.0 \%$ vs. $32.5-52.5 \%$; or enemas $50.0-71.4 \%$ vs. $23.3-50.0 \%$. For symptoms of constipation such as stool consistency and straining, lubiprostone treatment resulted in statistically significant improvements compared to placebo at each study week $(p \leq 0.001)$. In particular, lubiprostone statistically significantly improved overall stool consistency among patients who previously used contact laxatives $(p \leq 0.001)$, enemas $(p \leq 0.007)$ or PEG solutions $(p \leq 0.003)$ at all treatment weeks. Statistically significant improvements in straining were seen at all treatment weeks in patients who previously used PEG solutions $(\mathrm{p} \leq 0.018)$.

Conclusion Given the statistically significant response to lubiprostone in patients refractory to other constipation therapies, lubipro- stone may be a helpful addition to the armamentarium for patients suffering from chronic idiopathic constipation.

Disclosure of Interest None Declared.

\section{REFERENCE}

1. Müller-Lissner et al. Aliment Pharmacol Ther 2013; 37(1):137-145.

\section{PTH-196 LUBIPROSTONE DEMONSTRATES EFFICACY IN ADULT PATIENTS WITH CONSTIPATION REGARDLESS OF AGE, GENDER OR RACE}

doi:10.1136/gutjnl-2013-304907.683

'R M Panas, ${ }^{2, *} T$ Joswick, ${ }^{2} \mathrm{G}$ Dolecek, ${ }^{3 P}$ Lichtlen, 'D Panigrahi, ${ }^{4} \mathrm{R}$ Ueno. 'Medical Affairs; ${ }^{2} R \& D$, Sucampo Pharmaceuticals, Bethesda, United States; ${ }^{3} R \& D ;{ }^{4}$ Sucampo Pharmaceuticals, Zug, Switzerland

Introduction Constipation is a common condition affecting millions globally. Lubiprostone has demonstrated safety and efficacy in treating adults with chronic idiopathic constipation. Through an analysis of the subgroups of two Phase 3 studies, the efficacy of lubiprostone based on factors such as age, gender, and race was reviewed.

Aims and Methods Combined data from two Phase 3 well-controlled studies were used to analyse the following subpopulations: non-elderly ( $<65$ years) and elderly ( $\geq 65$ years); male and female; and white and non-white. The efficacy endpoints for spontaneous bowel movement (SBM) frequency, stool consistency, and straining were compared between placebo and lubiprostone (24 mcg BID) groups within each subpopulation.

Results Among non-elderly patients, lubiprostone produced a greater increase $(p \leq 0.001)$ in SBM frequency each week over placebo. Statistically significant increases were observed in the elderly $(p=0.0188$ to $p=0.0268)$ at each week except Week $2(p=0.0806)$. Statistically significant improvements were noted in females $(p \leq 0.001)$ at all weeks and in males at all weeks ( $p=0.0115$ to $p=0.0500)$ except Week $3(p=0.0758)$. For non-whites $(p=0.0003$ to $p=0.0021)$ and whites $(p \leq 0.001)$, statistically significant increases in SBM frequencies were reported at all weeks. Table 1 shows the weekly change in SBM frequency for each group. Stool consistency was improved with lubiprostone in the non-elderly ( $p=0.0172$ to $p=0.0441)$ and the elderly $(p \leq 0.001)$ at all weeks. Straining was also improved with lubiprostone treatment at all weeks in non-elderly patients $(p \leq 0.001)$, with similar improvement trends observed in the elderly. Stool consistency and straining were statistically significantly improved with lubiprostone at all weeks $(p \leq 0.001)$ in females. A similar trend was observed for males but did not achieve statistical significance at all weeks. Lubiprostone improved stool consistency at all weeks in whites $(p \leq 0.001)$ and non-whites $(p \leq 0.0001$ to $p=0.0006)$. Straining was improved at all weeks for whites $(p \leq 0.001)$ and non-whites $(p \leq 0.0001$ to $p=0.0144)$.

Table 1. Weekly Chenge in SBM Frequency

\begin{tabular}{|c|c|c|c|c|c|c|c|c|c|c|}
\hline & \multicolumn{9}{|c|}{} & \multicolumn{9}{c|}{ Mean Change From Baseline } \\
\hline & \multicolumn{2}{|c|}{ Baseline } & \multicolumn{2}{|c|}{ Weok 1 } & \multicolumn{2}{c|}{ Weok 2 } & \multicolumn{2}{c|}{ Wook 3 } & \multicolumn{2}{c|}{ Woøk 4 } \\
\hline & Plac & Lubi & Plac & Lubi & Plac & Lubi & Plac & Lubi & Plac & Lubi \\
\hline Non Elderly & 1.52 & 1.40 & 2.13 & 4.33 & 1.80 & 3.56 & 1.60 & 3.95 & 1.66 & 3.84 \\
\hline Elderly & 1.83 & 0.98 & 2.38 & 5.37 & 1.85 & 4.41 & 1.16 & 4.80 & 1.34 & 5.15 \\
\hline Malos & 1.28 & 1.38 & 2.13 & 4.77 & 1.20 & 4.66 & 1.91 & 4.91 & 1.47 & 4.31 \\
\hline Fomalos & 1.59 & 1.36 & 2.17 & 4.39 & 1.88 & 3.51 & 1.50 & 3.91 & 1.64 & 3.92 \\
\hline Non Whitos & 1.49 & 1.23 & 2.20 & 5.94 & 1.82 & 4.82 & 1.95 & 5.31 & 2.30 & 5.39 \\
\hline Whitos & 1.57 & 1.39 & 2.15 & 4.11 & 1.81 & 3.40 & 1.44 & 3.76 & 1.45 & 3.66 \\
\hline
\end{tabular}


Conclusion Treatment with lubiprostone resulted in increased SBM frequencies and improvement in related symptoms in patients with chronic idiopathic constipation regardless of age, gender, or race.

Disclosure of Interest None Declared.

\section{PTH-197 LONG-TERM EFFICACY OF LUBIPROSTONE DEMONSTRATED IN PATIENTS WITH CONSTIPATION REGARDLESS OF AGE, GENDER OR RACE}

doi:10.1136/gutjnl-2013-304907.684

1."T Joswick, 'G Dolecek, ${ }^{2} \mathrm{P}$ Lichtlen, ${ }^{3} \mathrm{D}$ Panigrahi, ${ }^{4} \mathrm{R}$ Ueno. ${ }^{1} R \& D$, Sucampo Pharmaceuticals, Bethesda, United States; ${ }^{2} R q D$, Sucampo Pharmaceuticals, Zug, Switzerland; ${ }^{3}$ Medical Affairs, Sucampo Pharmaceuticals, Bethesda, United States; ${ }^{4}$ Sucampo Pharmaceuticals, Zug, Switzerland

Introduction Constipation affects millions globally and may require chronic treatment. In 24- and 48-week trials, lubiprostone demonstrated safety and efficacy in long-term treatment of adults with chronic idiopathic constipation. Analyses of lubiprostone's (24 mcg BID) efficacy in age, gender and race subgroups were performed.

Aims and Methods Pooled data from three Phase 3 open-label safety studies were reviewed to analyse efficacy in the following subpopulations: non-elderly ( $<65$ years) and elderly ( $\geq 65$ years); male and female; and non-white and white. Constipation severity, abdominal bloating, and abdominal discomfort were rated on a 5 -point scale ranging from absent $=0$, mild $=1$, moderate $=2$, severe $=3$ and very severe $=4$, and changes from baseline following treatment with lubiprostone were analysed.

Results In the non-elderly and elderly, lubiprostone improved constipation severity $(p \leq 0.0001)$ each week beginning at Week 1 and continuing through Week 48. Among males and females, lubiprostone improved ( $p \leq 0.0001)$ constipation severity each week with similar findings $(p \leq 0.0001)$ for non-whites and whites, For abdominal discomfort, significant changes were seen at all weeks in the non-elderly $(p \leq 0.0001)$ and for all weeks in the elderly $(p \leq 0.0001$ to $p=0.0150)$ except Week $8(p=0.0530)$. Among the genders, improvements occurred at all weeks in males $(p \leq 0.0001$ to $p=0.0220)$ and in females $(p \leq 0.0001)$. By race, improvements occurred at all weeks among nonwhites $(p \leq 0.0001$ to $p=0.0020)$ and among whites $(p \leq 0.0001)$. Significant changes in abdominal bloating were seen in the non-elderly $(p \leq 0.0001)$ and for the elderly $(p \leq 0.0001$ to $p=0.0180)$ at all weeks. Abdominal bloating improved at all weeks for males ( $p \leq 0.0001$ to $p=0.0010)$ and females $(p \leq 0.0001)$. For non-whites and whites, abdominal bloating improved $(p \leq 0.0001)$ at all weeks.

\section{Abstract PTH-197 Table 1 Change from Baseline for Constipation Severity}

\begin{tabular}{llcccccc}
\hline & & \multicolumn{6}{c}{ Mean Change From Baseline } \\
\hline \multirow{3}{*}{ Age } & & Baseline & Week 1 & Week 12 & Week 24 & Week 36 & Week 48 \\
& $<65$ Years & 2.91 & -1.21 & -1.18 & -1.21 & -1.19 & -1.14 \\
\multirow{3}{*}{ Gender } & $\geq 65$ years & 2.76 & -1.11 & -0.97 & -1.00 & -1.15 & -1.03 \\
& Male & 2.65 & -1.21 & -1.12 & -1.18 & -1.27 & -1.17 \\
& Female & 2.92 & -1.19 & -1.14 & -1.17 & -1.16 & -1.11 \\
Race & Non-Whites & 2.98 & -1.52 & -1.51 & -1.52 & -1.48 & -1.38 \\
& Whites & 2.87 & -1.14 & -1.08 & -1.12 & -1.13 & -1.08 \\
\hline
\end{tabular}

Conclusion Lubiprostone demonstrated long-term efficacy through an overall improvement in constipation severity for up to 12 months regardless of age, gender, or race. Similarly, improvements were noted in abdominal bloating and abdominal discomfort for both race and gender subpopulations along with some significant and positive trend for improvement in the age group analyses. Disclosure of Interest None Declared.

\section{PTH-198 POOLED ANALYSIS OF THE MOST FREQUENT ADVERSE EVENTS ASSOCIATED WITH THE USE OF LUBIPROSTONE}

doi:10.1136/gutjnl-2013-304907.685

1. ${ }^{*} \mathrm{~T}$ Joswick, ${ }^{1} \mathrm{G}$ Dolecek, ${ }^{2 P}$ Lichtlen, ${ }^{3} \mathrm{D}$ Panigrahi, ${ }^{4} \mathrm{R}$ Ueno. ${ }^{1} R \notin D$, Sucampo Pharmaceuticals, Bethesda, United States; ${ }^{2} R \& D$, Sucampo Pharmaceuticals, Zug, Switzerland; ${ }^{3}$ Medical Affairs, Sucampo Pharmaceuticals, Bethesda, United States, ${ }^{4}$ Sucampo Pharmaceuticals, Zug, Switzerland

Introduction Lubiprostone, a novel $\mathrm{ClC}-2$ activator, has been shown to be efficacious and well-tolerated by patients with chronic constipation in short- and long-term clinical trials. To better characterise the most frequent adverse events (AEs) associated with the use of lubiprostone $24 \mathrm{mcg} \mathrm{BID}$, we examined pooled results of patients enrolled in Phase 2 and 3 trials of 3 to 48 weeks' duration. Methods Data for all safety-evaluable patients were pooled and compared between treatment groups (placebo vs lubiprostone 24 mcg BID). Nausea, headache, and diarrhoea AEs were analysed in terms of severity, duration, frequency, action taken (eg, drug withdrawn, dose reduced), and outcome. In addition, nausea-related variables were explored in subpopulations of elderly ( $\geq 65$ years of age) and male patients.

Results The pooled population included 1113 lubiprostone and 316 placebo patients. Approximately 16\% were elderly and 13\% were male. At least $1 \mathrm{AE}$ occurred in $79.1 \%$ of lubiprostone patients, which included $31.1 \%$ with nausea, $13.2 \%$ with headache, and $13.2 \%$ with diarrhoea. At least $1 \mathrm{AE}$ occurred in $39.6 \%$ of placebo patients, which included $5.1 \%$ with nausea, $6.6 \%$ with headache, and $0.9 \%$ with diarrhoea. Of those lubiprostone patients who experienced nausea, $88.7 \%$ reported nausea to be mild or moderate in severity. Overall, $74.5 \%$ of nausea events reported by lubiprostone patients were intermittent, and the median durations of nausea events were 12 and 7.5 days for lubiprostone and placebo patients, respectively. Notably, mean incidences of nausea per patient were 1.3 and 1.0 for lubiprostone and placebo patients, respectively. With regard to action taken, $64.1 \%$ of nausea events required no change in treatment regimen, and $9.6 \%$ of events resulted in dose reduction. Nausea was less prevalent in subpopulations, occurring in only $18.8 \%$ and $8.2 \%$ of lubiprostone elderly and male patients, respectively, compared with $34.5 \%$ of female patients. Similarly, the majority of lubiprostone patients experiencing headache and diarrhoea had mild-to-moderate symptom severity $(89.8 \%$ and $82.3 \%$, respectively). Furthermore, headache and diarrhoea events in lubiprostone patients were mostly intermittent (64.2\% and $74.0 \%$, respectively), and the median duration of these events were 9 and 4 days, respectively. Discontinuation rates due to nausea, headache, and diarrhoea were $8.7 \%, 3.7 \%$, and $2.2 \%$, respectively.

Conclusion Nausea, headache, and diarrhoea associated with lubiprostone use are generally mild to moderate in severity, intermittent, and limited in duration.

Disclosure of Interest None Declared.

\section{PTH-199 PREVALENCE OF ORGANIC DISORDERS IN CONSECUTIVE NEW PATIENTS MEETING CRITERIA FOR IBS IN A GASTROENTEROLOGY CLINIC}

doi:10.1136/gutjnl-2013-304907.686

1.*U Shivaji, 'D Gracie, 'A Sainsbury, 'A C Ford. 'Gastroenterology, Leeds Teaching Hospitals, Leeds, UK

Introduction A positive diagnosis of irritable bowel syndrome (IBS), without the need for recourse to investigation, is encouraged. Patients meeting symptom-based diagnostic criteria for IBS are often given reassurance that there is no serious underlying pathology, and treated symptomatically. However, some studies have suggested that an organic diagnosis, such as coeliac disease or pancreatic 